\title{
Hip-spine relations and sagittal balance clinical consequences
}

\author{
Jean-Yves Lazennec $\cdot$ Adrien Brusson • \\ Marc-Antoine Rousseau
}

Received: 11 July 2011/Accepted: 11 July 2011/Published online: 28 July 2011

(C) The Author(s) 2011. This article is published with open access at Springerlink.com

\begin{abstract}
Introduction The role of the pelvic area in sagittal balance is evident for spinal surgeons, but the influence of the coxofemoral joint is underestimated and inadequately explained by conventional imagery. Comprehensive analysis of the pelvic and subpelvic sectors as part of the sagittal, frontal and cross-sectional balance of the trunk sheds new light on some spinal diseases and their relation to the pelvis.

Methods This analysis, based on innovative radiologic methods as the $\operatorname{EOS}^{\circledR}$ technology but also on a new look at conventional imaging makes it possible to better analyze standing lateral images and seated images.

Results Disturbances can come from atypical morphotypes or from unusual postures as in aging spine. The measurement of available extension and the concept of available flexion provide new information regarding individual's adaptation to the imbalance induced by disorders of the spine or lower limbs.

Conclusion A comprehensive assessment of each patient and in particular of the complex comprising the spine and the pelvis, is essential for understanding each individual's adaptation to the imbalance induced by disorders of the spine or lower limbs.
\end{abstract}

J.-Y. Lazennec $(\bowtie) \cdot$ M.-A. Rousseau

Service de chirurgie orthopédique, Hôpital Pitié Salpêtrière, Université Pierre et Marie Curie, Assistance Publique-Hôpitaux de Paris, Paris 6, 83 bd de l'hôpital, 75013 Paris, France e-mail: lazennec.jy@wanadoo.fr

A. Brusson

Ingénieur de recherches cliniques, Hôpital Pitié Salpêtrière, Assistance Publique-Hôpitaux de Paris, Paris, France
Keywords Sagittal balance - Incidence angle . Hip prosthesis impingement - Hip range of motion . Hip prosthesis dislocation - EOS imaging - Acetabular anteversion - Anterior pelvic plane $\cdot$ Pelvic tilt

\section{Introduction}

Trunk balance is the manifestation of a postural strategy conditioned by anatomic and functional characteristics, sometimes very different from one person to another. Degeneration of this steady state, often associated with spinal aging, can generate a cascade of functional, neurological, and mechanical events. The role of the pelvic area in sagittal balance is evident for spinal surgeons, who consider sacral slope, pelvic incidence, and pelvic tilt in their planning and analyses [1-6]. On the other hand, the influence of the coxofemoral joint is underestimated and inadequately explained by conventional imagery. These anatomic and functional considerations often remain foreign to hip surgeons, who focus on the bone landmarks of the pelvis for their navigation, individualized adjustments, and failure analyses. The AP or frontal image of the pelvis is the "gold standard"; a lateral view of the pelvic area is rarely used. Influenced by the classic anatomic culture of cross-sectional slices, they consider the CT scan as a reference tool for the "horizontal" assessment of the coxofemoral joints [5-10].

Our objective is to call attention to a broader view of the pelvic and subpelvic regions as part of the sagittal, AP and transversal balance of the trunk. This analysis, based on innovative radiologic methods as the $\operatorname{EOS}^{\circledR}$ technology but also on an original approach of conventional imaging makes it possible to look with new eyes at numerous aspects of diseases of the hip and spine, from the perspective of a real functional entity. 


\section{Fundamental principles: postural variations of the lumbopelvic bone markers in the sagittal plane}

Each subject is characterized by a "morphologic" parameter: the angle of incidence, which schematically represents pelvic thickness. The adaptation of other functional factors, such as pelvic tilt, and the spinal parameters (sacral slope, lumbar lordosis, and thoracic kyphosis) makes it possible to position the center of gravity of the trunk specifically so that it is supported by the femoral heads relative to the pelvic base, to maintain balance with only a minimal muscular effort [11-13] (Fig. 1).

The pelvis moves, rotating around the bicoxofemoral axis, leading to both anterior tilt (where the upper portion of the pelvis tips forward) and posterior tilt (upper portion of the pelvis tips backward). The variations in the angles of the sacral slope determine the range of this pelvic tilt, and its variations in turn influence the orientation of the anterior pelvic plane or the Lewinnek plane classically used as a reference for navigation of the acetabular components in total hip arthroplasties (THA) (Fig. 2) [14]. The surprising consequences of some spinal arthrodesis or stiffening on the hips and the difficulties of adjusting prostheses below stiffened spines illustrate the importance of these anatomicfunctional parameters.

The standing position (Fig. 3) corresponds to a forward tilt of the pelvis as a whole. In this situation, the upper plate of S1, viewed laterally, makes an angle of approximately 35 to $45^{\circ}$ between the sacral slope and the horizontal $[15,16]$. Some subjects have a small sacral slope angle in standing position: we talk then about posterior pelvic tilt (or pelvic retroversion or pelvic extension), and the sacrum seen on a lateral image appears more vertical than usual (Fig. 4).

On the contrary, other subjects have a very horizontal sacrum in standing position with a sacral slope angle

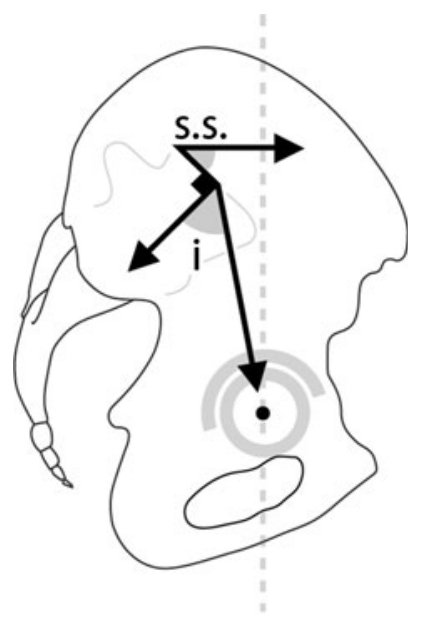

Fig. 1 Definition of the pelvic incidence (morphologic parameter invariant for a given subject, with a mean value of $50-55^{\circ}$ ) and of the sacral slope (functional parameter that varies according to position)
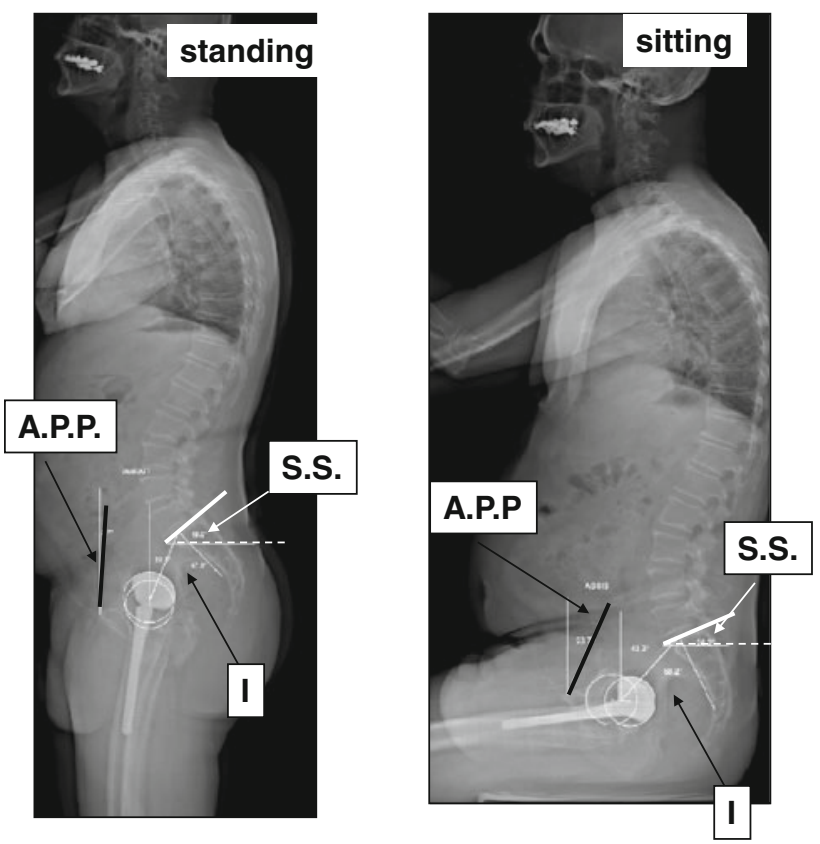

Fig. $2 \operatorname{EOS}^{\circledR}$ views of the sagittal balance of a patient with a THA, in standing and seated positions. The incidence does not change regardless of position. The sacral slope is higher in a standing than a seated position. Simultaneous variations of the inclination of Lewinnek's plane (the posterior tilt of this plane is associated with the posterior pelvic tilt in a seated position). $I$ incidence, $S S$ sacral slope, $A P P$ angle between the Lewinnek plane and the vertical plane

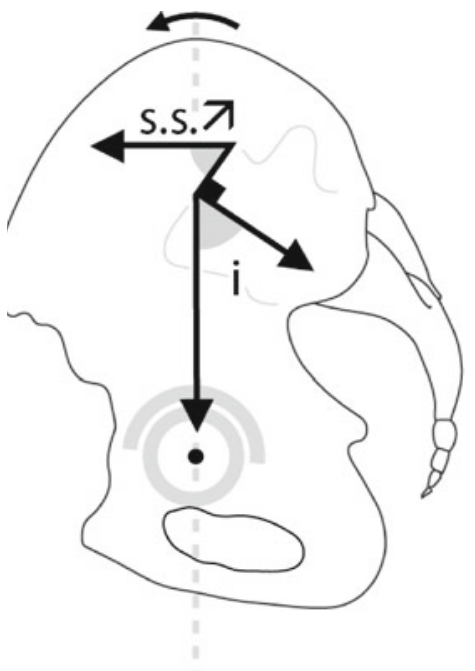

STANDING

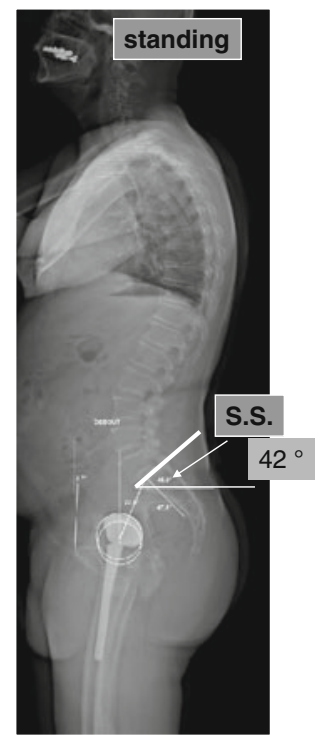

Fig. 3 Diagram of the standing position: anterior tilt (flexion) of the pelvis with horizontalization of the sacrum and increase of the sacral slope. The incidence is invariable and does not change

sometimes much greater than $50^{\circ}$ (anterior pelvic tilt or pelvic anteversion or pelvic flexion) (Fig. 5).

In the supine position, when the lower limbs are extended, the sacral slope is often greater than in standing position. The measurement of its angle with the vertical 
Fig. 4 Permanent posterior pelvic tilt and imbalance of a spine stiffened by a long arthrodesis. The sacral slope is low in both the standing and seated positions. In standing position, the pelvis and the spine function as if the patient were in a seated position
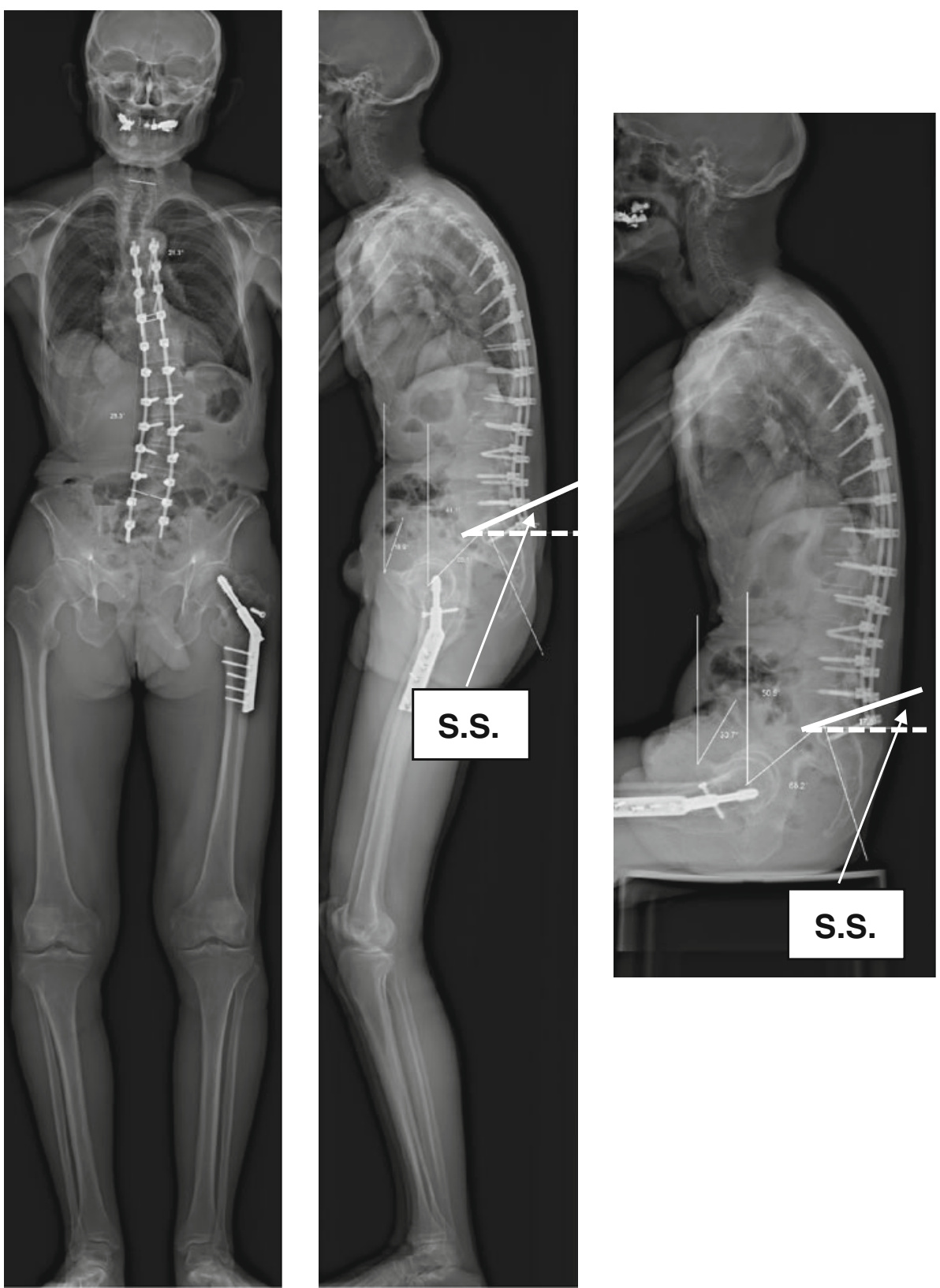

often exceeds $45^{\circ}[17,18]$. This can be seen on scout views of pelvic CT scan. This additional pelvic tilt may not be well tolerated in the case of a stiff or deformed spine since the dorsal decubitus position deepens lumbar lordosis. Sometimes a limitation of the available extension of the hips or posterior osteoarthritis make a strict decubitus position unbearable, at least without a slight compromise flexion of the coxofemoral joints.

In a sitting position, the phenomenon is inversed (Fig. 6). The pelvis tilts backwards as it progresses toward a sitting position. The sacral slope diminishes, to values of 20 to $25^{\circ}$ on average $[15,17]$. This slope may still be slightly positive (by $5-10^{\circ}$ ) or even sometimes negative. As a function of the height of the seat, the subject's morphology or any associated spinal disease, we observe pelvic posterior tilt (pelvic retroversion or pelvic extension) more or less accentuated with a sacrum more or less vertical. The difference in the values of the sacral slope between standing and seated positions makes it possible to define the available flexion associated with the lumbosacral joint (extrinsic available pelvic flexion) by opposition to the potential for flexion associated with the coxofemoral joint (intrinsic available pelvic flexion).

Progression from standing to sitting positions causes considerable modification in the orientation of the anterior pelvic plane, today considered the reference for adjusting the acetabular cups [19-21]. These variations must concern us. Lewinnek's plane (anterior pelvic plane) is evaluated in 
Fig. 5 Patient with an exaggerated anterior pelvic tilt: the sacrum is very horizontal in standing position with a sacral slope greater than normal
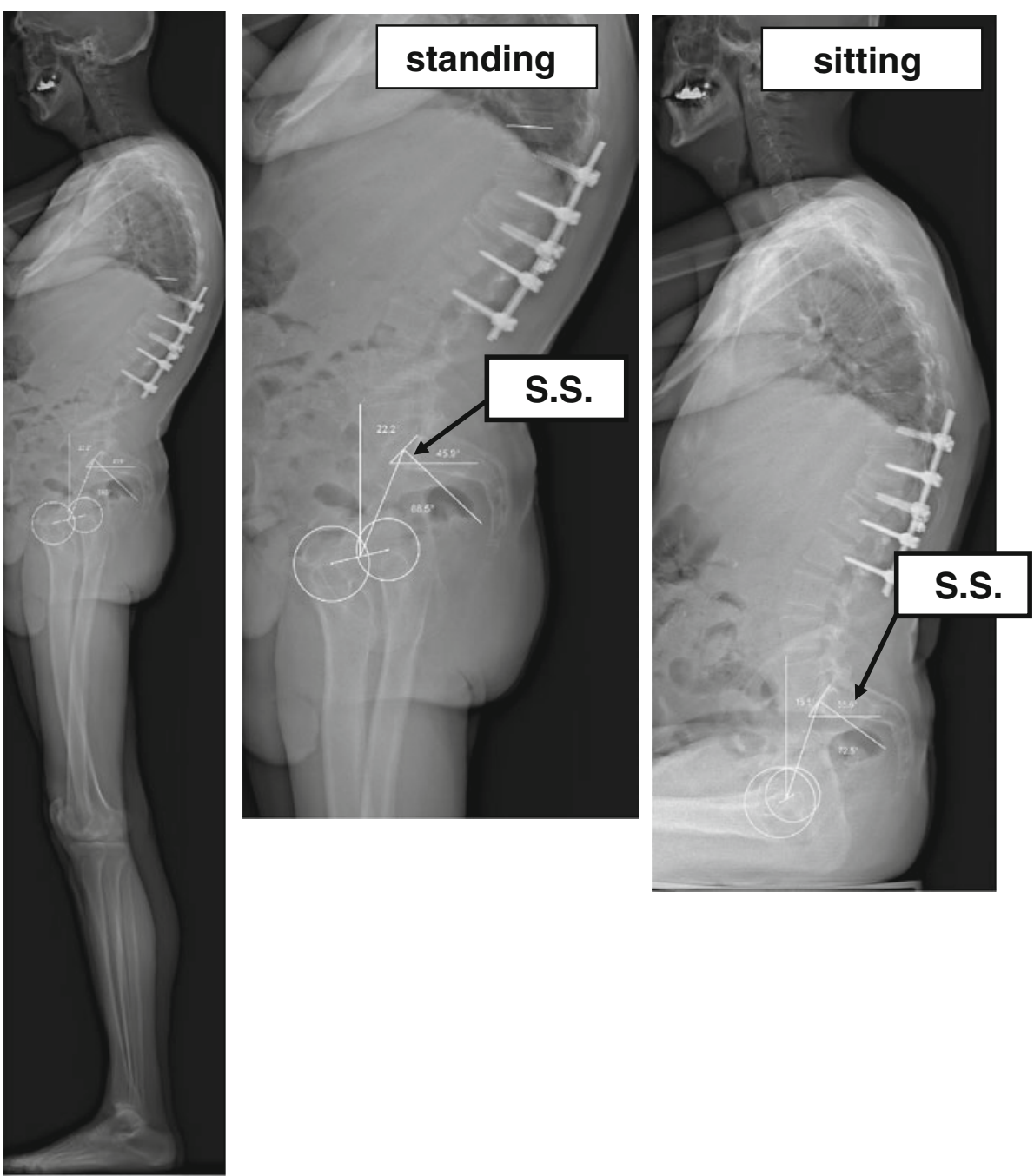

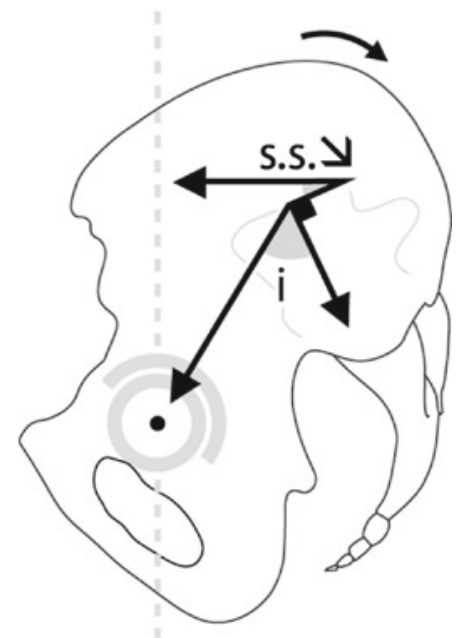

SITTING

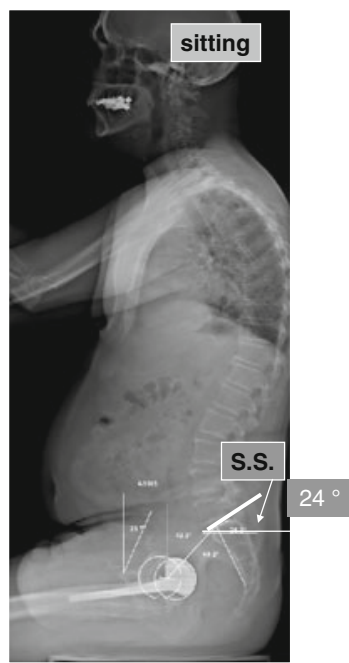

Fig. 6 Diagram of the seated position: posterior pelvic tilt with verticalization of the sacrum and decrease of the sacral slope. The incidence is invariable and does not change the dorsal decubitus position to collect morphologic data for planning the hip prosthesis [14]. The information obtained must extrapolated prudently because it is not necessarily vertical in standing positions, and its tilt is highly variable in sitting positions [22]. Accordingly, for mean values of $3^{\circ}$ in standing positions, the values reported while sitting were $17.5^{\circ}$ [15] (Fig. 7).

\section{Influence of sagittal posture on the frontal and sagittal acetabular orientation in functional situations}

\section{Anatomic foundations}

The interdependence between the sagittal tilt of the cup and the sacral slope is obvious when reading lateral images, seated or standing, of the lumbosacral joint [23].

In a standing position, the value of the sacral slope is high and the angle of the acetabular tilt is small. Inversely, 
a
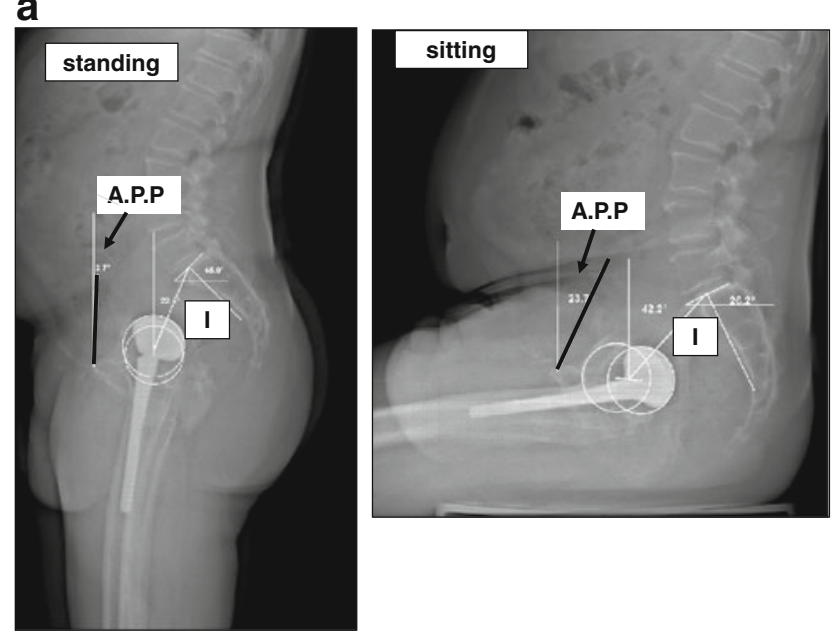

b
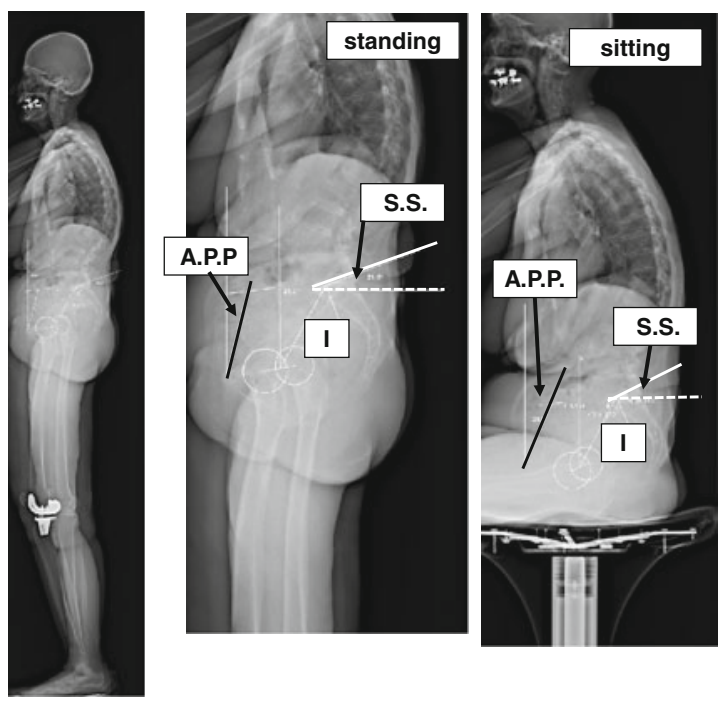

Fig. 7 Variations in sagittal balance from the standing to the seated position. a Usual variation: The anterior pelvic plane tilts backwards in a seated position at the same time that the sacral slope is reduced from $45^{\circ}$ standing to $26^{\circ}$ seated; in this case, the available pelvic

in a seated position, the sacral slope diminishes and the acetabular tilt increases. From both AP and lateral positions, the THA cup appears more vertical in seated than in standing positions. Observational series of THA from diverse institutions $[15,17]$ report mean values of 49 to $52^{\circ}$ for the frontal cup inclination in standing position and 57 to $64^{\circ}$ while seated. At the same time the sagittal inclination is 36 to $47^{\circ}$ standing and 51 to $58^{\circ}$ seated. These variations in acetabular tilt contribute to modifying the "anterior opening" of the acetabulum and therefore the orientation of the functional "mobility cone" generated by the coxofemoral joint (Fig. 8) [17, 24].

Modifications in the acetabular orientation produce consequences for the stability of hip arthroplasties and for

\section{C}

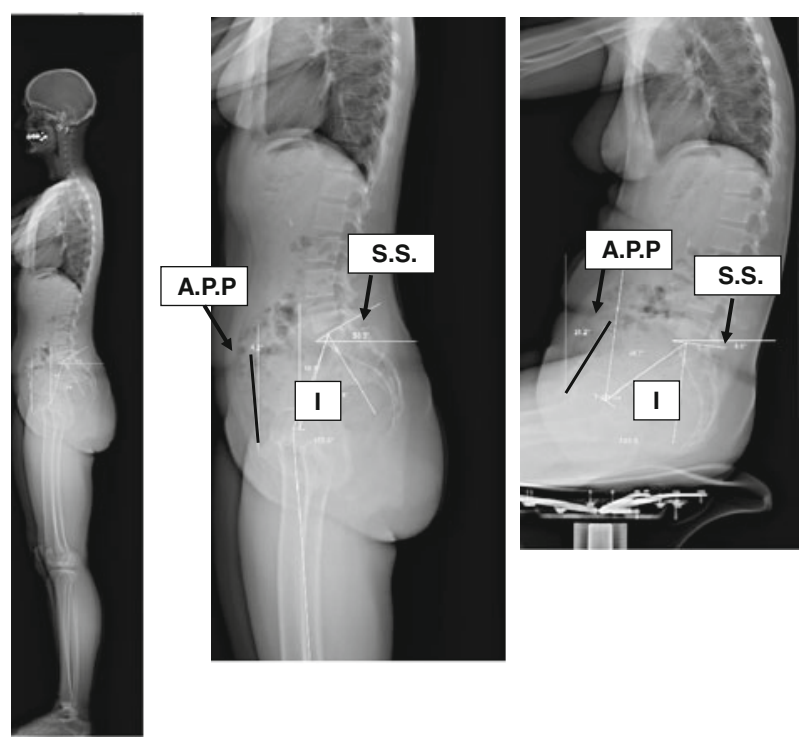

flexion is $19^{\circ}$. b Little postural variation of a stiffened lumbosacral joint (no available pelvic flexion). c Strong postural variations, sacral slope negative in a seated position (available pelvic flexion $38^{\circ}$ )

all aspects of their tribology in the intermediate and long term [25-29].

\section{Disruptions can come from atypical postures}

Excess posterior tilt of the pelvis is often associated with postural imbalance to compensate for a forward tilt of the entire trunk. This postural adaptation verticalizes the cup from both the AP and lateral views and places the hips in a hyperextended position when standing. This solicitation of the available hip extension can lead to a posterior impingement. A typical example is posterior impingement of the hip prosthesis in standing position even though the cup was placed perfectly in accordance with the pelvic 


\section{standing}
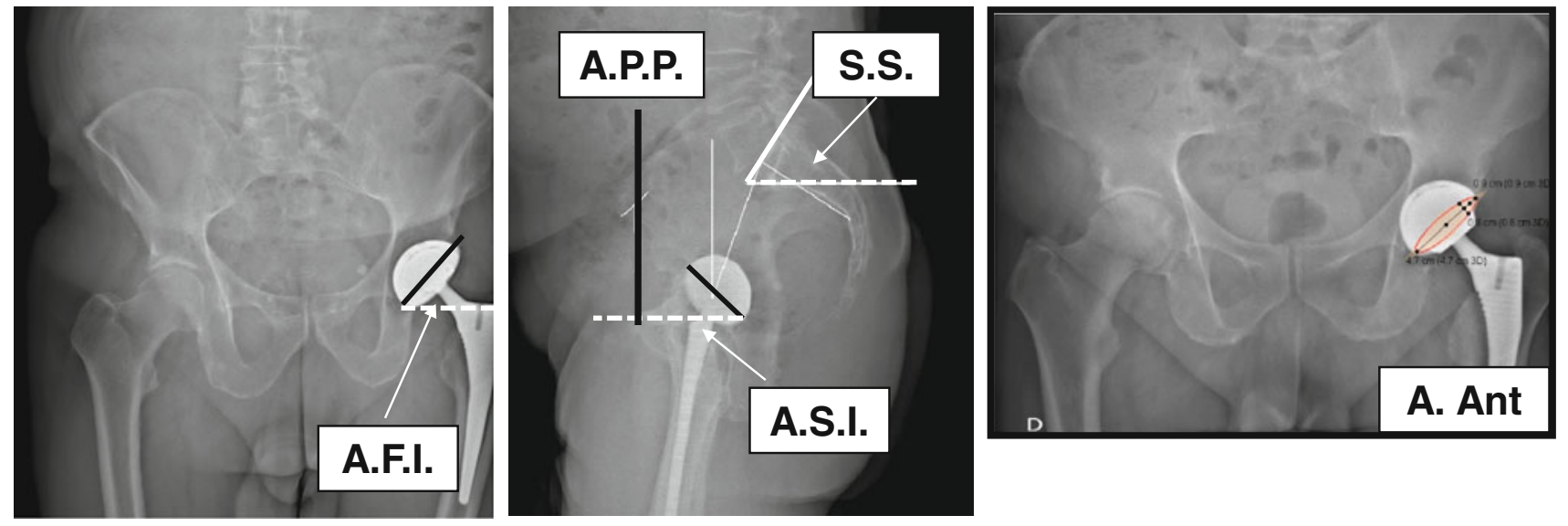

\section{sitting}
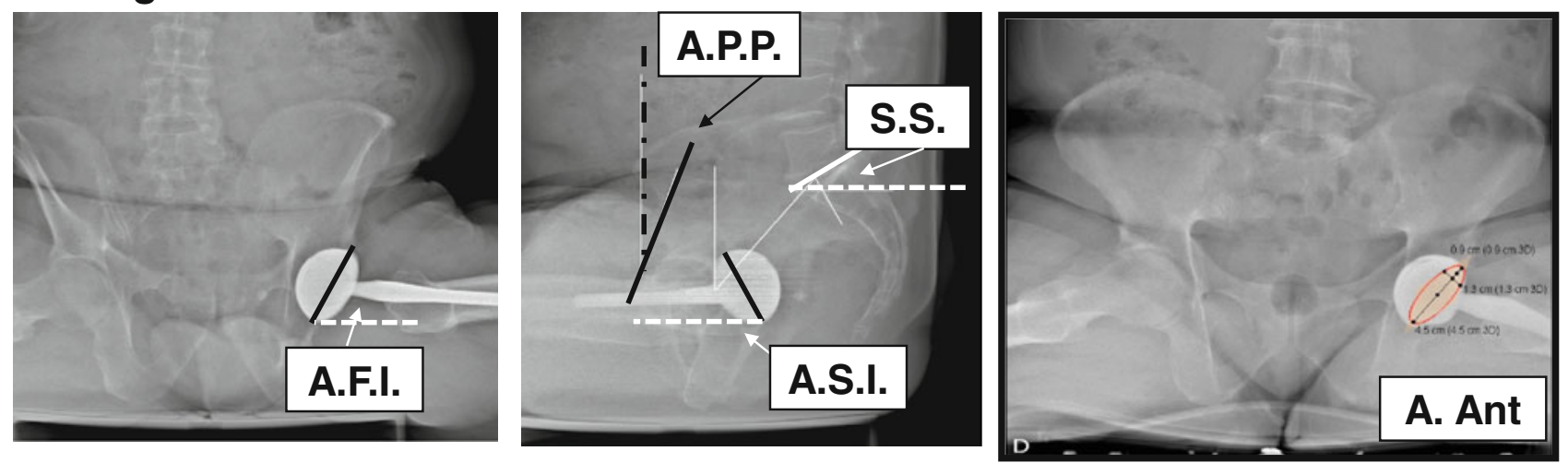

Fig. 8 Modification of the acetabular orientation from standing to seated position: increase in the acetabular frontal inclination (AFI.), the acetabular sagittal inclination (ASI), and the acetabular anterior opening (A Ant) measured by radiologic anteversion (Pradhan)

bone landmarks (Fig. 9). This situation can be encountered in native hips in the case of excessive posterior tilt of the pelvis (abnormal posture, trunk aging and induced posterior coxarthrosis) [30-33]. Analysis of these situations can be difficult because they involve simultaneously diseases of the spine and hips. Assessment of the available hip extension is thus essential to distinguish true and false inability to extend the hip fully (Figs. 10,11). This analysis can be performed easily with EOS $^{\circledR}$ technology, which makes it possible to individualize the available extension associated with the lumbosacral joint, that is, the extrinsic available extension, as well as the intrinsic available extension of each coxofemoral joint.

Inversely, excess anterior tilt of the pelvis in a seated position can cause anterior impingement (Fig. 12). In some cases, the anterior tilt of the acetabulum is also excessive in a standing position: the acetabulum is "horizontalized" from both AP and lateral views, as if the hips are permanently flexed when the subject is standing. This type of mechanism is suggested on native hips in anterior impingements, especially in some repetitive athletic or occupational movements.

We also observed a mechanism of specific adaptation in hips that could not be fully extended secondary to coxarthrosis. The loss of range of motion of the abnormal hip results in a forward tilt of the pelvis when the patient tries to straighten up. When possible, the spine adapts by increasing lumbar lordosis, thus causing low back pain. Frequently, only one hip is involved. The test for available extension allows us to assess the phenomenon selectively.

\section{Disturbances can come from atypical morphotypes}

The angle of incidence is a morphologic parameter determinant for the adaptation of sagittal spinopelvic balance. It is associated with 2 functional indicators, pelvic tilt and sacral slope, by the formula $\mathrm{I}=\mathrm{SS}+\mathrm{PT}$.

In subjects with a high angle of pelvic incidence, theoretical lumbar lordosis is greater as the range of adaptation 
Fig. 9 The unusual posterior tilt of the pelvis can be detected on the AP standing image by the overly visible foramina obturata. After THA, this could result in a posterior impingement in standing position (anterior dislocation or subluxation)
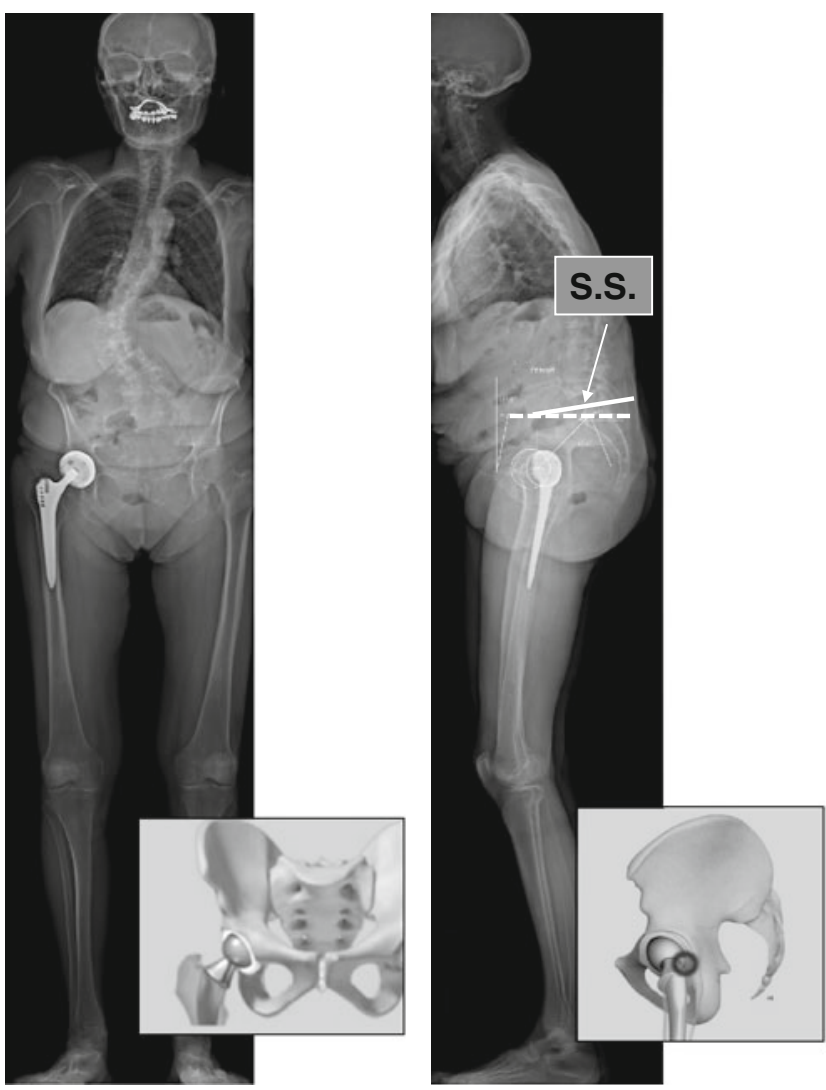

of the sacral slope may also be. The femoral heads are projected forward relative to the sacrum and the acetabular anterior opening is less marked. The coxofemoral joints of these subjects have a greater theoretical available extension and therefore a better ability to adapt.

Inversely, in subjects with a low pelvic incidence angle, there is less theoretical lumbar lordosis and the adaptability of the sacral slope may be more limited. The femoral heads are embedded under the sacrum and the anterior opening of the acetabulum is more marked. Theoretically, these subjects have less available extension and a weaker capacity to adapt to sagittal imbalance (Fig. 13).

\section{Influence of sagittal posture on cross-sectional acetabular cup orientation: anatomic and functional anteversion}

\section{Standard data}

"Anatomic" anteversion or "morphological" anteversion is the angle between the AP pelvic axis and the acetabular axis when this is projected on to the transverse reference plane perpendicular to the longitudinal axis and the mid sagittal plane of the pelvis [6].
CT scan measurements are supposed to assess anatomic anteversion directly from cross-sectional slices. But the orientation of these slices in relation to the sagittal plane is left to radiologists to assess without any specific criterion for standardization, although it has a critical effect on the values of the angles measured. Fortuitously, they can be strictly perpendicular to the longitudinal axis of the pelvic bone landmarks. Most of the time these slices, perpendicular to the plane of the examination table, do not correspond to the anatomic plane because the position of the supine subject has a more or less marked sagittal pelvic tilt [17]. The variation in the anteversion measured is approximately 0.5 for $1^{\circ}$ of rotation of the plane of the slice in relation to the pelvis $[18,34,35]$.

An accurate understanding of lumbosacral posture and its influence on cup tilt shows us that the measurement of anteversion must not be linked to an arbitrary orientation of the slices to the machine stand. This point is essential for assessing the real acetabular anteversion of THA in terms of instability, especially when the lumbosacral joint is stiffened or in an atypical position [36-38].

The concept of functional anteversion

Functional acetabular anteversion is measured by the angle of the anterior opening of the cup, which varies as a 

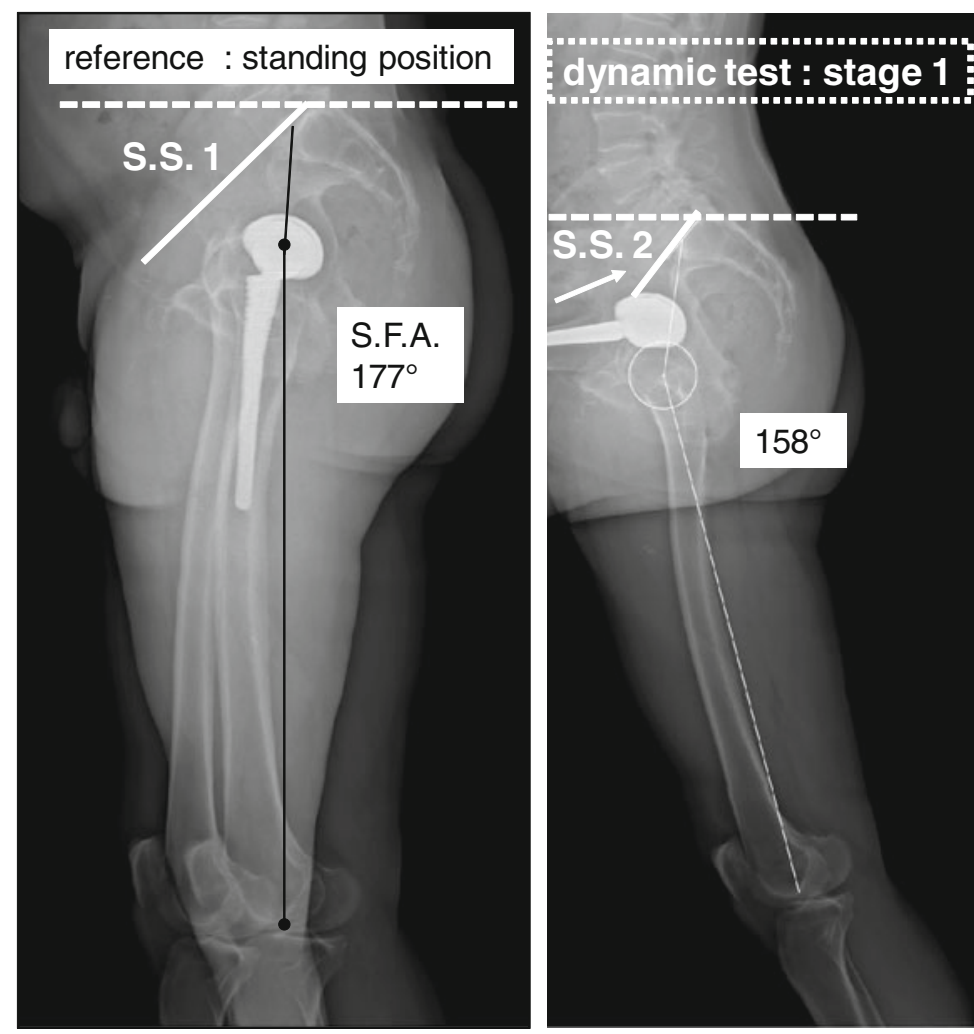

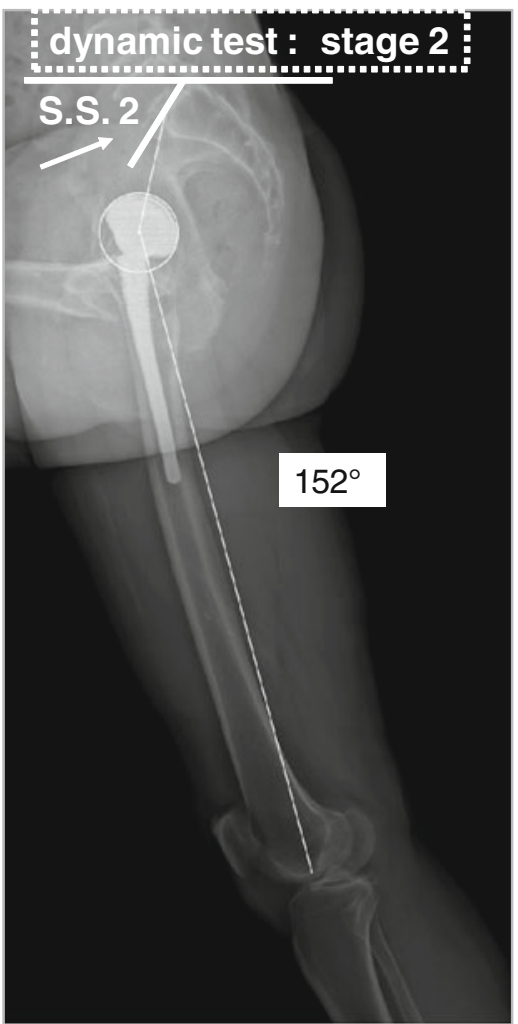

Fig. 10 Principle of measurement of the available spinopelvic extension $\left(\right.$ EOS $^{\circledR}$ system). a The patient is assessed in a lateral standing position. (b, c) Each hip is then assessed in its maximum range of extension in standing position, placing the contralateral lower limb on a step such that it is in a position of maximum flexion of the coxofemoral joint. The extrinsic available extension is measured by the capacity to increase the sacral slope (SS 2-SS 1). The intrinsic available extension is measured by the variation of the

function of pelvic tilt; it is calculated from the CT scan slices taken as a function of the sacral slope measured on the initial standard lateral image.

In a plane with the upper sacral plate, these slices reconstitute the sacral tilt angle, measured in a standing, sitting, or supine position. They make it possible to observe important changes in the cup orientation and improve our understanding of some coxofemoral joint dysfunctions.

In standing positions, there is less cross-sectional anteversion than in sitting positions, where the cup opens completely forward, thus favoring flexion of the hip and avoiding impingement at the femoral neck (Fig. 14). In supine positions, with the lower limbs extended, the pelvic tilt is often greater than in standing positions, lordosis more accentuated and anteversion still more reduced than when standing. In a series of 328 THA, we reported a mean anteversion of $31.7^{\circ}$ standing, $38.8^{\circ}$ seated, and $24.2^{\circ}$ supine [17].

Globally, the "standard" CT scan measurements tend to overestimate anteversion in standing positions and to underestimate it in seated positions. Some subjects have sacro-femoral angle (SFA). The sacro-femoral angle is defined as the angle between the segment drawn from the middle of the sacral plate to the middle of the segment joining the centers of the femoral heads, and the segment joining the latter point (midpoint of the segment joining the center of the femoral heads) to the summit of the Blumensaat line, at the knee. The intrinsic extension is $177-$ $152^{\circ}=25^{\circ}$ for the THA side and $177-158^{\circ}=19^{\circ}$ for the nonoperated hip

completely stiff lumbosacral joints, and this significantly reduces the variations in acetabular anteversion between seated and standing positions. Such stiffening of the pelvis, in either a relative anterior or posterior tilt, may lead to a reproducible impingement situation. This phenomenon has been documented in the literature for deformities of the sagittal plane in spondylarthritis, in poorly adjusted lumbosacral arthrodesis (flat back), and especially in spinal aging [30-32, 39-41]. In these cases, we observed a reduction in lordosis and the appearance of an adaptive posterior pelvic tilt. The patient is standing, as if he were seated. This phenomenon reduces adaptation in the lumbosacral area and deviates the functional mobility cone of the coxofemoral joints towards flexion. The cup is permanently in hyper-anteversion, which is not bothersome during hip flexion but creates a problem of posterior impingement, especially in standing position: the person progressively loses his or her available extension (Fig. 15). The compensating hyperextension of the hips often has a limited impact in these patients, especially those who are elderly or have hip disorders. The last adaptation to attempt 
a

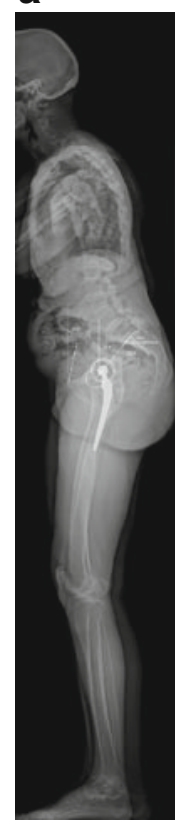

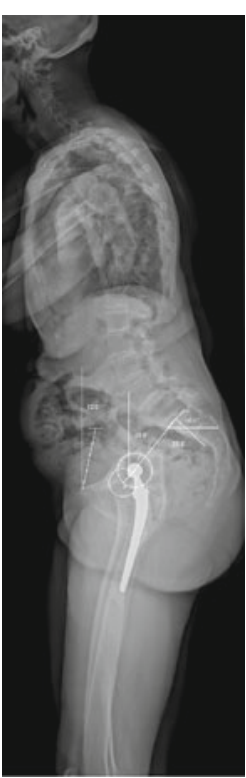

\section{b}

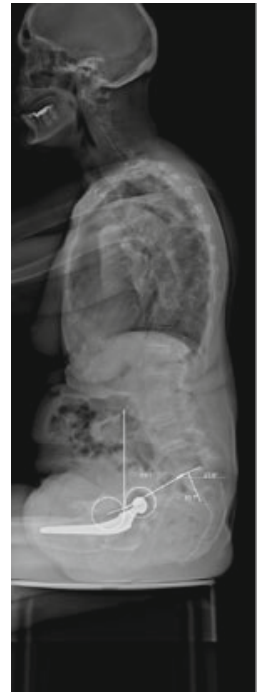

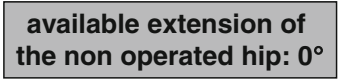
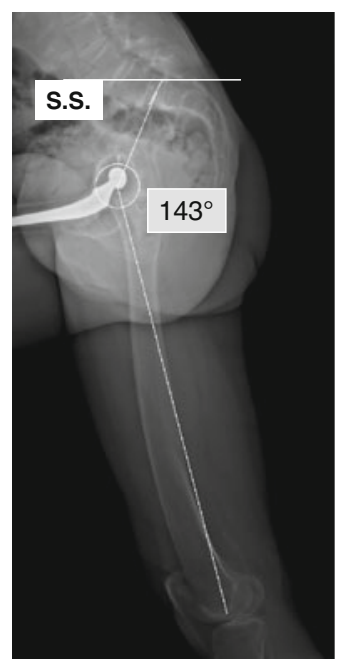
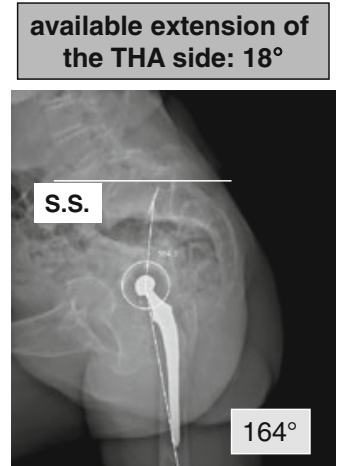

Fig. 11 a Situation of spinopelvic imbalance and hips that do not extend completely. The incidence is high; it is theoretically associated with elevated lordosis to keep the sagittal balance stable. In this case, the patient has an anterior imbalance not compensated by the hips (absence of hyperextension). This imbalance can be analyzed by searching for the available extension. b No role for the spine in recovering the extension: no available extrinsic extension because there is no modification of the sacral slope. There is no available extension for the non-operated hip (full extension truly impossible). Only the hip that underwent THA has an increase in available extension: from $164-146^{\circ}=18^{\circ}$. The presence of this available extension on the operated side is evidence of an adaptive false inextendability in the reference standing position
Fig. 12 The abnormal anterior tilt of the pelvis in a seated position can lead to an anterior impingement of the hips with the risk of lesions of the labrum and the cartilage. In these cases, the available hip flexion required by the seated position is exaggerated, as the pelvic posterior tilt (pelvic retroversion) is not sufficient
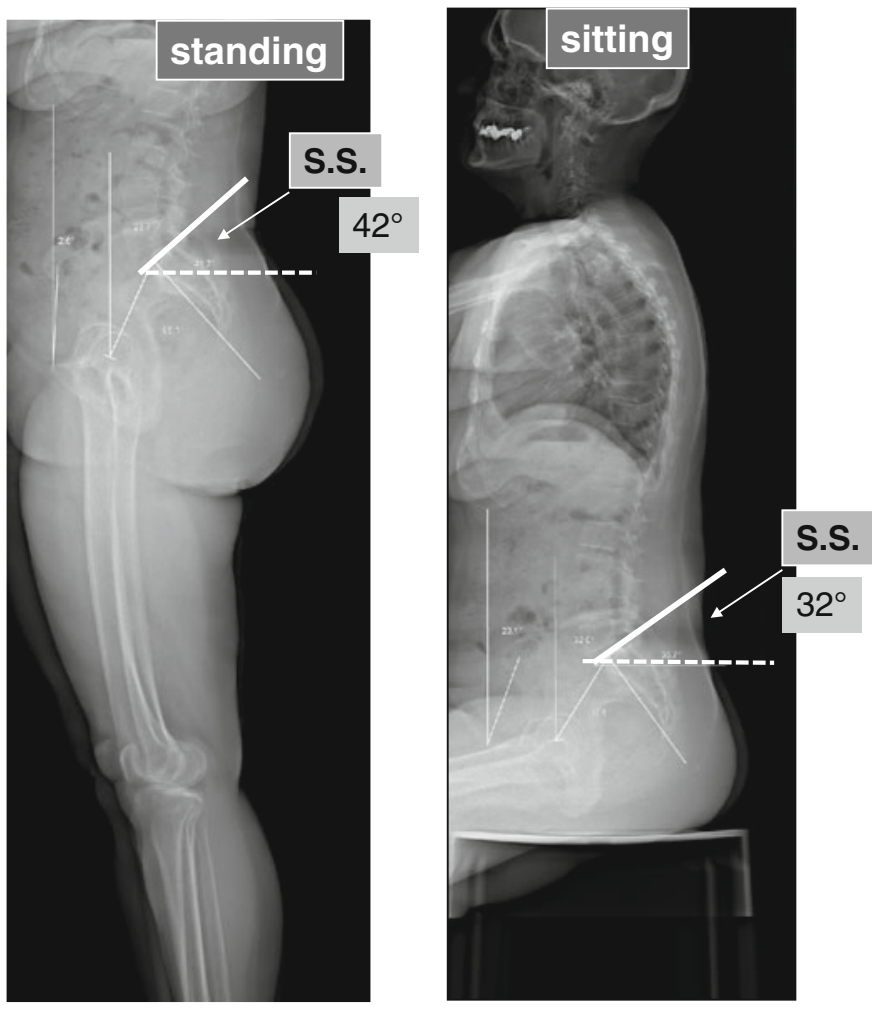

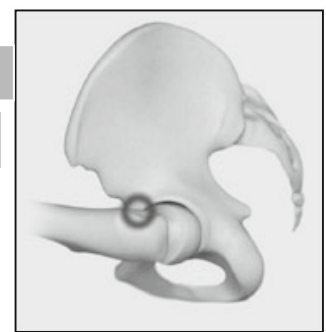




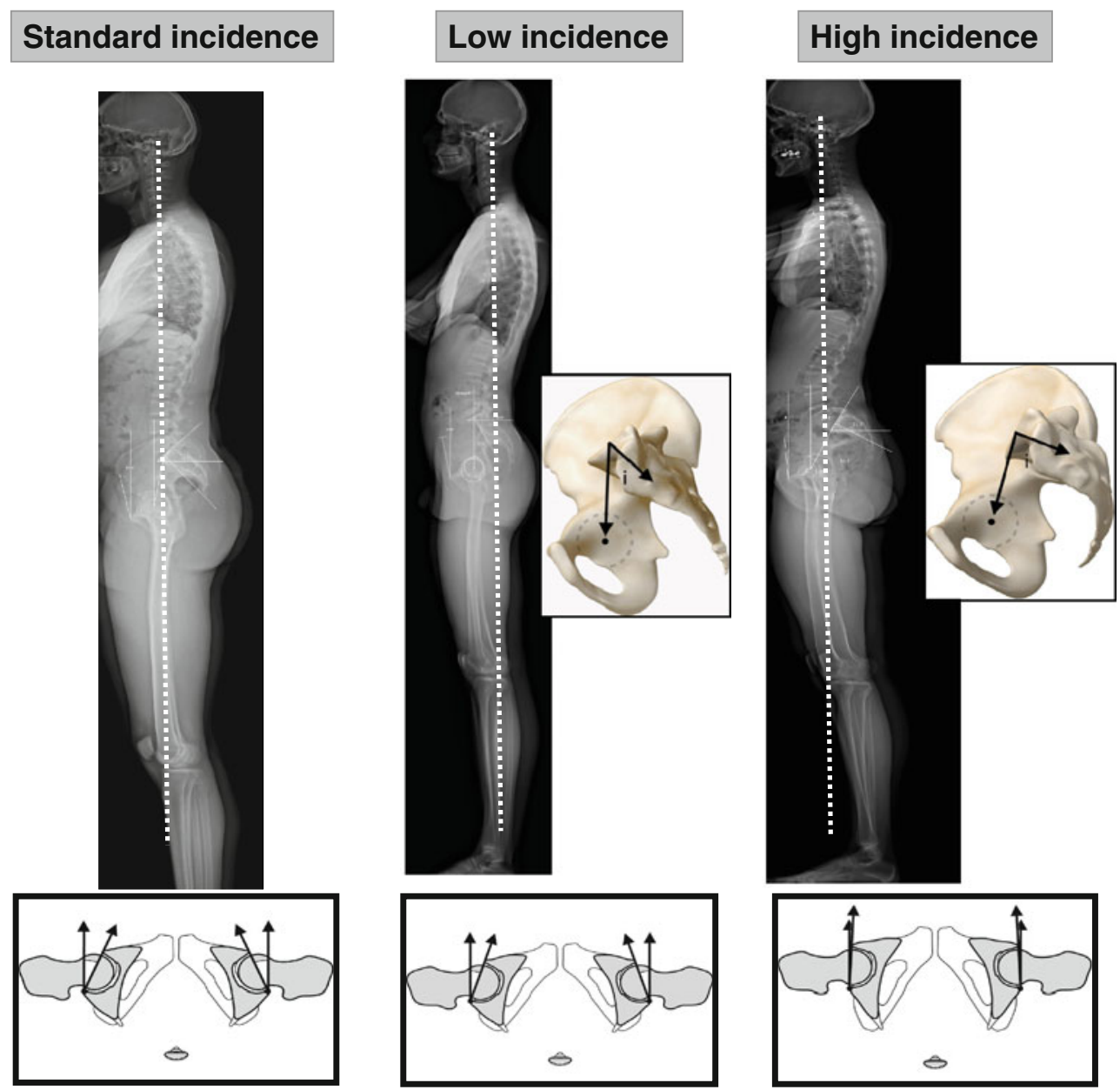

Fig. 13 Incidence is a morphologic characteristic determinant in the adaptation of the sagittal spinopelvic balance. In subjects with a high angle of pelvic incidence, lumbar lordosis is greater and the range of adaptation of the sacral slope may be greater. The anterior opening of the acetabulum is less marked, and the available extension of the hips

to improve balance is thus flexion of the knees, which enables additional posterior tilt of the pelvis.

\section{Influence of axial rotation of the pelvis and the lumbopelvic region}

Hip surgeons define a "normal" pelvic posture for imaging work-ups of subjects in a strictly anatomic position, with the two iliac wings projecting exactly and symmetrically, compared with the longitudinal axis of the trunk. Spine surgeons have been made aware of the threedimensional view of the spine and the phenomenon of vertebral rotation that disturbs analysis of the lateral view. In this framework, the concept of the pelvic vertebra leads us to integrate pelvic rotation into the analysis of the overall trunk posture [42]. greater. In the case of low pelvic incidence, lumbar lordosis is lower and the range of adaptation of the sacral slope more limited. The anterior opening of the acetabulum is more marked, but the theoretically available extension is lower at the hips

The use of standing and seated $\operatorname{EOS}^{\circledR}$ images in the subject's "usual" position is particularly instructive. Our database of complete $\operatorname{EOS}^{\circledR}$ acquisitions, both standing and seated, from over 2,500 patients, reveals the frequency of cases involving a forward hemipelvis and therefore a backward contralateral hemipelvis. This is expressed on the AP image by asymmetry of the projection of the iliac wings; the "forward" wing appears thinner than the other. Laterally, the two femoral heads and the two iliac wings are not superimposed. This "twisting" phenomenon is difficult to quantify with standard radiography because of the cone-shaped distribution of X-rays, which distorts interpretation of the image of the femoral head furthest from the scanner source. On the other hand, it is well analyzed by $\operatorname{EOS}^{\circledR}$ images in both the standing and seated positions, and 3D visualization of the pelvic position is possible. This 
Fig. 14 In standing position, the sacral slope is high $\left(40^{\circ}\right.$ approximately); the acetabulum appears more horizontal from both frontal and lateral views; the functional acetabular anteversion is weaker. In a seated position, the sacral slope is lower, sometimes negative, and the acetabulum appears more vertical from both the frontal and lateral views. The functional acetabular anteversion is more elevated
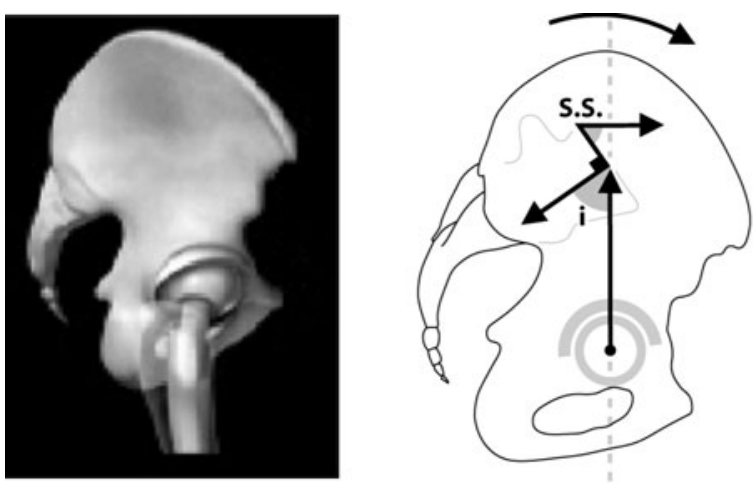

STANDING
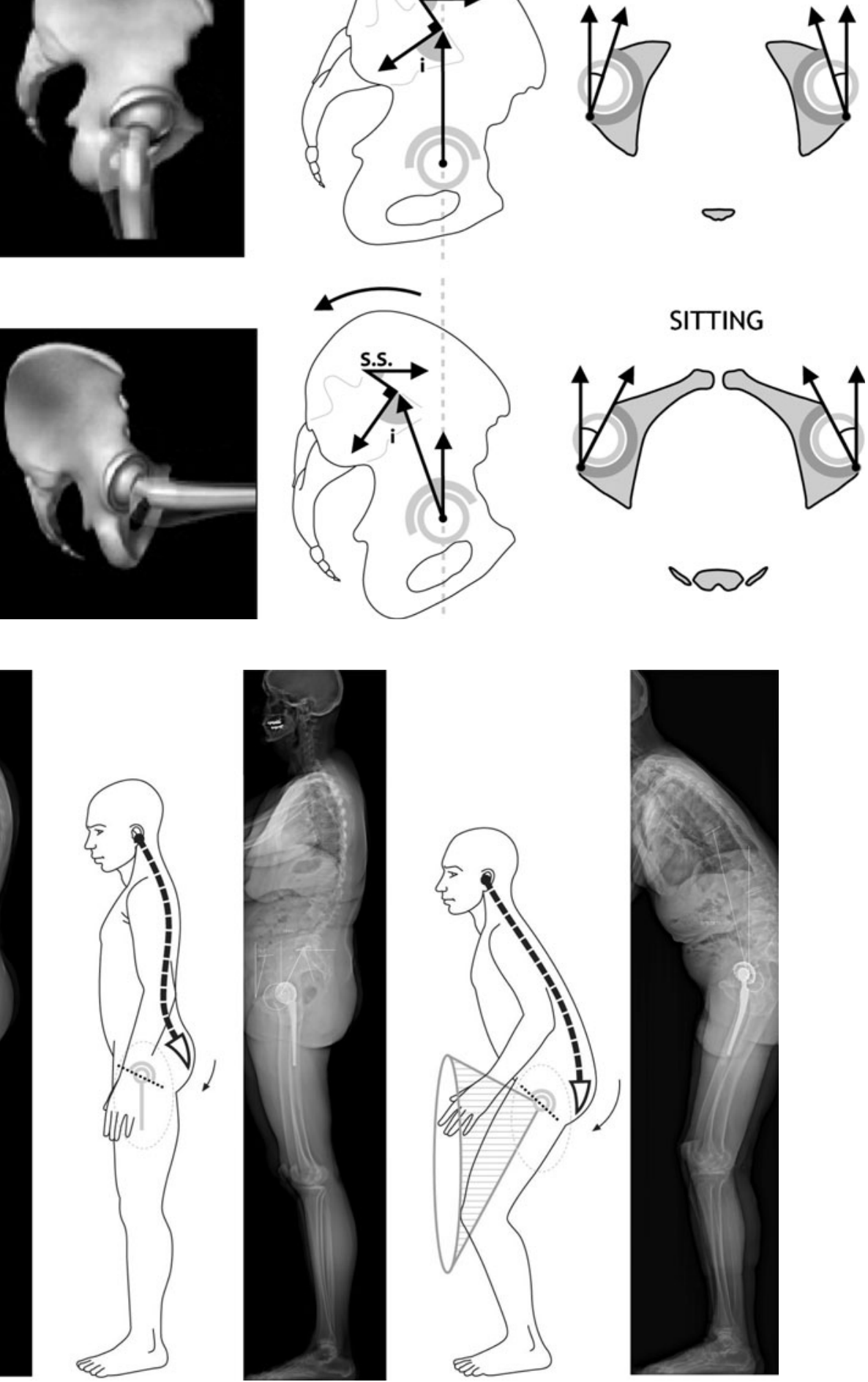

Fig. 15 Modifications of the functional mobility cone in cases of pelvic tilt disorders: in the case of a posterior tilt of the pelvis, the cone is deflected; even though the coxofemoral joints are perfectly mobile, the patient is using his available extension

situation of pelvic rotation is pushed to extremes in cases of scoliosis with the pelvic vertebrae included in the deformity (Fig. 16). The consequences on cup orientation 

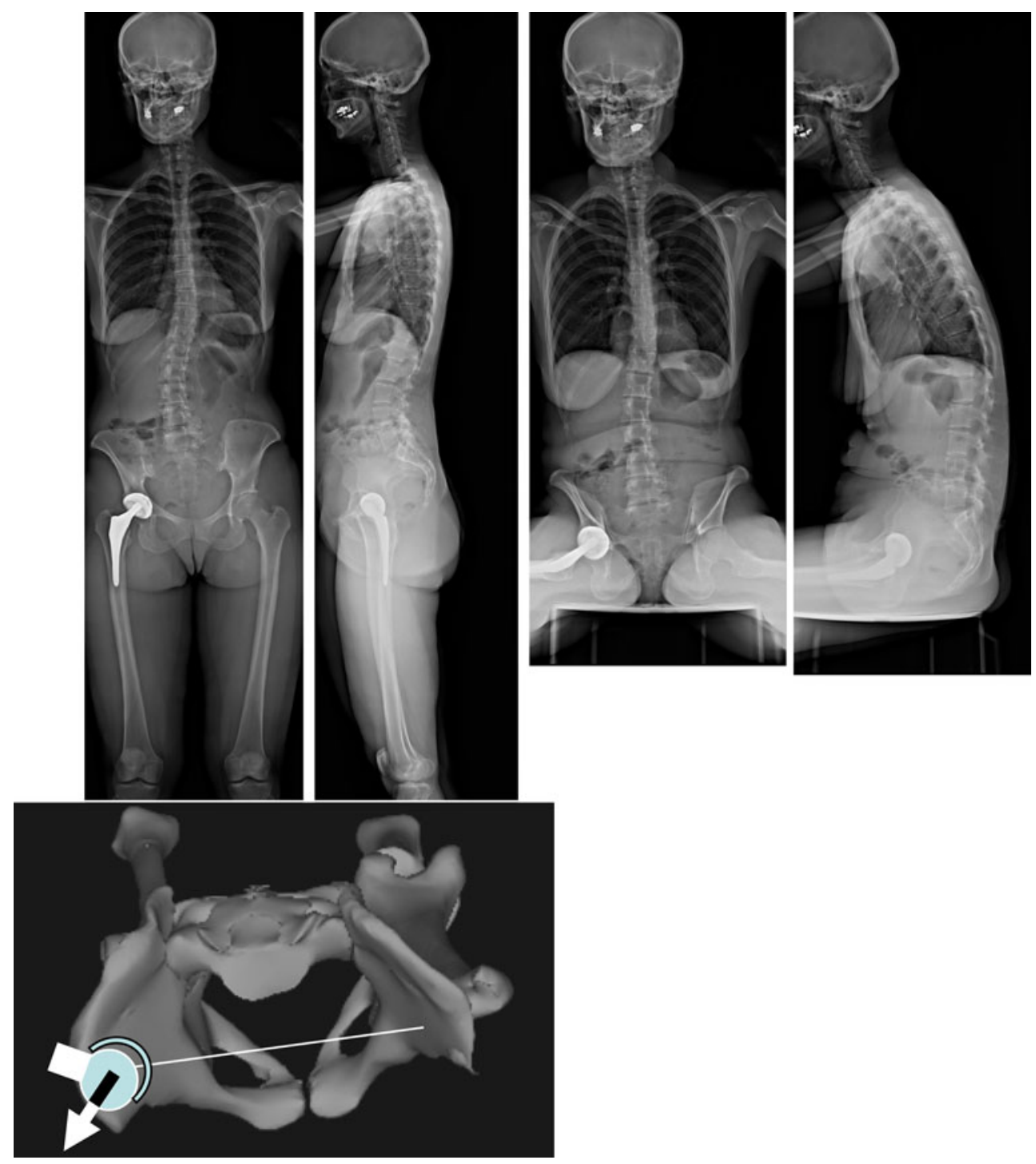

Fig. 16 Substantial rotation of the pelvis associated with scoliosis: the THA is perfectly implanted relative to the bone landmarks but subluxated permanently forward in the standing position: pelvic

\section{Conclusion}

Comprehensive analysis of the pelvic and subpelvic sectors as part of the sagittal, frontal and cross-sectional balance of the trunk sheds new light on some spinal diseases and their relation to the pelvis.

Knowledge of the mechanics of the lumbosacral joint is essential both for the spine surgeon and for the surgeon implanting hip replacements in elderly subjects or those with abnormal sagittal, frontal or rotational posture and/or a large reduction in functional range of motion.

Analysis of sagittal trunk balance must not therefore stop at the simple analysis of standing lateral spine images. The position of the hips is essential and the interpretation rotation induced an abnormal acetabular anteversion in a standing position. Note that the pelvic rotation disappears in the seated position (patient is asymptomatic when sitting)

of hips that do not extend completely must integrate the measurement of available extension. Seated images show us large variations in balance and lead us to take into account the concept of available flexion, which is essential in hip surgery and in situations of instability.

A comprehensive assessment of each patient and in particular of the complex comprising the spine, pelvis, and femur is essential for understanding each individual's adaptation to the imbalance induced by disorders of the spine or lower limbs. Monitoring the course of these patients and planning treatment strategies, surgical or not, can thus be rationalized and optimized.

\section{Conflict of interest None.}


Open Access This article is distributed under the terms of the Creative Commons Attribution Noncommercial License which permits any noncommercial use, distribution, and reproduction in any medium, provided the original author(s) and source are credited.

\section{References}

1. Kobayashi $\mathrm{T}$ et al (2004) A longitudinal study of congruent sagittal spinal alignment in an adult cohort. Spine (Phila Pa 1976) 29(6):671-676

2. Chanplakorn $P$ et al (2011) Lumbopelvic alignment on standing lateral radiograph of adult volunteers and the classification in the sagittal alignment of lumbar spine. Eur Spine J 20(5):706-712

3. Vialle R et al (2005) Radiographic analysis of the sagittal alignment and balance of the spine in asymptomatic subjects. J Bone Joint Surg Am 87(2):260-267

4. Jackson RP, McManus AC (1994) Radiographic analysis of sagittal plane alignment and balance in standing volunteers and patients with low back pain matched for age, sex, and size. A prospective controlled clinical study. Spine (Phila Pa 1976) 19(14):1611-1618

5. Padgett DE, Warashina $H$ (2004) The unstable total hip replacement. Clin Orthop Relat Res 420(1):72-79

6. Murray DW (1993) The definition and measurement of acetabular orientation. J Bone Joint Surg Br 75(2):228-232

7. Kennedy JG et al (1998) Effect of acetabular component orientation on recurrent dislocation, pelvic osteolysis, polyethylene wear, and component migration. J Arthroplasty 13(5):530-534

8. Ackland MK, Bourne WB, Uhthoff HK (1986) Anteversion of the acetabular cup. Measurement of angle after total hip replacement. J Bone Joint Surg Br 68(3):409-413

9. Seki M, Yuasa N, Ohkuni K (1998) Analysis of optimal range of socket orientations in total hip arthroplasty with use of computeraided design simulation. J Orthop Res 16(4):513-517

10. Wan $\mathrm{Z}$ et al (2009) Imaging and navigation measurement of acetabular component position in THA. Clin Orthop Relat Res 467(1):32-42

11. Duval-Beaupere G, Schmidt C, Cosson P (1992) A Barycentremetric study of the sagittal shape of spine and pelvis: the conditions required for an economic standing position. Ann Biomed Eng 20(4):451-462

12. Roussouly P et al (2005) Classification of the normal variation in the sagittal alignment of the human lumbar spine and pelvis in the standing position. Spine (Phila Pa 1976) 30(3):346-353

13. Legaye $\mathbf{J}$ et al (1998) Pelvic incidence: a fundamental pelvic parameter for three-dimensional regulation of spinal sagittal curves. Eur Spine J 7(2):99-103

14. Lewinnek GE et al (1978) Dislocations after total hip-replacement arthroplasties. J Bone Joint Surg Am 60(2):217-220

15. Lazennec JY et al (2011) Pelvis and total hip arthroplasty acetabular component orientations in sitting and standing positions: measurements reproductibility with EOS imaging system versus conventional radiographies. Orthop Traumatol Surg Res 97(4):373-380

16. Lazennec JY et al (2000) Sagittal alignment in lumbosacral fusion: relations between radiological parameters and pain. Eur Spine J 9(1):47-55

17. Lazennec JY et al (2011) Acetabular anteversion with CT in supine, simulated standing, and sitting positions in a THA patient population. Clin Orthop Relat Res 469(4):1103-1109

18. Lazennec JY et al (2004) Hip-spine relationship: a radio-anatomical study for optimization in acetabular cup positioning. Surg Radiol Anat 26(2):136-144
19. D'Lima DD et al (2000) The effect of the orientation of the acetabular and femoral components on the range of motion of the hip at different head-neck ratios. J Bone Joint Surg Am 82(3):315-321

20. Rousseau MA et al (2009) Optimization of total hip arthroplasty implantation: is the anterior pelvic plane concept valid? J Arthroplasty 24(1):22-26

21. Herrlin K et al (1988) Position, orientation and component interaction in dislocation of the total hip prosthesis. Acta Radiol 29(4):441-444

22. Philippot $R$ et al (2009) Pelvic balance in sagittal and Lewinnek reference planes in the standing, supine and sitting positions. Orthop Traumatol Surg Res 95(1):70-76

23. Bolger $\mathrm{C}$ et al (2007) Electrical conductivity measurement: a new technique to detect iatrogenic initial pedicle perforation. Eur Spine J 16(11):1919-1924

24. Pradhan R (1999) Planar anteversion of the acetabular cup as determined from plain anteroposterior radiographs. J Bone Joint Surg Br 81(3):431-435

25. Yamaguchi M, Bauer TW, Hashimoto Y (1997) Three-dimensional analysis of multiple wear vectors in retrieved acetabular cups. J Bone Joint Surg Am 79(10):1539-1544

26. von Knoch $M$ et al (2002) Late dislocation after total hip arthroplasty. J Bone Joint Surg Am 84-A(11):1949-1953

27. Casanova $G$ et al (1985) The minifixator of the Army health service. Presentation and initial results. Apropos of 18 cases. Rev Chir Orthop Reparatrice Appar Mot 71(Suppl 2):102-104

28. Lazennec JY et al (2007) Hip spine relationships: application to total hip arthroplasty. Hip Int 17(Suppl 5):S91-S104

29. Woo RY, Morrey BF (1982) Dislocations after total hip arthroplasty. J Bone Joint Surg Am 64(9):1295-1306

30. Itoi E (1991) Roentgenographic analysis of posture in spinal osteoporotics. Spine (Phila Pa 1976) 16(7):750-756

31. Hammerberg EM, Wood KB (2003) Sagittal profile of the elderly. J Spinal Disord Tech 16(1):44-50

32. Offierski CM, MacNab I (1983) Hip-spine syndrome. Spine (Phila Pa 1976) 8(3):316-321

33. Lafage $\mathrm{V}$ et al (2009) Pelvic tilt and truncal inclination: two key radiographic parameters in the setting of adults with spinal deformity. Spine (Phila Pa 1976) 34(17):E599-E606

34. Anda $S$ et al (1990) Pelvic inclination and spatial orientation of the acetabulum. A radiographic, computed tomographic and clinical investigation. Acta Radiol 31(4):389-394

35. Muller $\mathrm{O}$ et al (2005) Quantification and visualization of the influence of pelvic tilt upon measurement of acetabular inclination and anteversion. Z Orthop Ihre Grenzgeb 143(1):72-78

36. Lembeck B et al (2005) Pelvic tilt makes acetabular cup navigation inaccurate. Acta Orthop 76(4):517-523

37. Terver S (1982) True orientation of the acetabulum as determined by CAT scan. Preliminary results (author's transl). J Radiol 63(3): 167-173

38. Eddine TA et al (2001) Variations of pelvic anteversion in the lying and standing positions: analysis of 24 control subjects and implications for CT measurement of position of a prosthetic cup. Surg Radiol Anat 23(2):105-110

39. During $\mathbf{J}$ et al (1985) Toward standards for posture. Postural characteristics of the lower back system in normal and pathologic conditions. Spine (Phila Pa 1976) 10(1):83-87

40. Fogel GR, Esses SI (2003) Hip spine syndrome: management of coexisting radiculopathy and arthritis of the lower extremity. Spine J 3(3):238-241

41. Lazennec JY et al (1997) Surgery of the deformities in ankylosing spondylitis: our experience of lumbar osteotomies in 31 patients. Eur Spine J 6(4):222-232

42. Dubousset J et al (2007) EOS stereo-radiography system: wholebody simultaneous anteroposterior and lateral radiographs with very low radiation dose. Rev Chir Orthop Reparatrice Appar Mot 93(6 Suppl):141-143 